\title{
The Relationship between Insufficient Sleep and Self-Rated Health in a Nationally Representative Sample
}

\author{
Sarah Dee Geiger, ${ }^{1}$ Charumathi Sabanayagam, ${ }^{1,2}$ and Anoop Shankar ${ }^{1}$ \\ ${ }^{1}$ Department of Community Medicine, West Virginia University School of Medicine, Morgantown, WV 26506, USA \\ ${ }^{2}$ Singapore Eye Research Institute and Department of Clinical Sciences, Duke-NUS Graduate Medical School, Singapore 169857
}

Correspondence should be addressed to Anoop Shankar, ashankar@hsc.wvu.edu

Received 16 November 2011; Accepted 22 February 2012

Academic Editor: David Strogatz

Copyright (C) 2012 Sarah Dee Geiger et al. This is an open access article distributed under the Creative Commons Attribution License, which permits unrestricted use, distribution, and reproduction in any medium, provided the original work is properly cited.

\begin{abstract}
Reduced sleep has been found to be associated with increased risk of diabetes mellitus, hypertension, cardiovascular disease (CVD), and mortality. Self-rated health (SRH) has been shown to be a predictor of CVD and mortality. However, study of the association between insufficient sleep and SRH is limited. We examined participants $>18$ years of age $(n=377,160)$ from a representative, cross-sectional survey (2008 BRFSS). Self-reported insufficient sleep in the previous 30 days was categorized into six groups. The outcome was poor SRH. We calculated odds ratios ((OR) (95\% confidence interval (CI)) of increasing categories of insufficient rest/sleep, taking zero days of insufficient sleep as the referent category. We found a positive association between increasing categories of insufficient sleep and poor SRH, independent of relevant covariates. In the multivariable-adjusted model, compared to 0 days insufficient sleep, the OR (95\% CI) of poor SRH was 1.03 (0.97-1.10) for 1-6 days, 1.45 (1.34-1.57) for 713 days, 2.12 (1.97-2.27) for 14-20 days, 2.32 (2.09-2.58) for 21-29 days, and and 2.71 (2.53-2.90) for 30 days of insufficient sleep in the prior 30 days $(P$-trend $<0.0001)$. In a nationally representative sample, increasing categories of insufficient sleep were associated with poor SRH.
\end{abstract}

\section{Introduction}

Sleep loss, long-term sleep deprivation, and insufficient rest/sleep are pervasive problems in developed countries today. Short sleep duration and insufficient rest/sleep may also be a manifestation of underlying sleep-disordered breathing (SDB). Reduced sleep has been found to be associated with increased risk of diabetes mellitus, hypertension, cardiovascular disease (CVD), and cardiovascular and allcause mortality [1-5]. Similarly, self-rated health (SRH) has been shown to be a consistent predictor of cardiovascular disease and cardiovascular and overall mortality [6-9]. Selfrated health $(\mathrm{SRH})$ is a commonly used subjective measure of health used in epidemiological studies as well as a secondary outcome in clinical trials to examine changes in subjective health status [6-10]. Epidemiological studies from several countries and social and cultural settings have shown that SRH is a valid and consistent predictor of CVD and cardiovascular and overall mortality [6-9]. Due to its predictive ability of future risk of mortality as well as objective health outcomes, an Institute of Medicine (IOM) report in 2001 recommended the inclusion of SRH measurement in national surveys in the USA as a way of tracking the subjective health status of Americans [11].

However, the mechanisms underlying the reported association between SRH and CVD and mortality are not known. It is possible that at least part of the association between insufficient sleep and mortality may be mediated by poor SRH-although, to our knowledge, no study has examined this topic. In this context, we examined association between insufficient rest/sleep and SRH in a large multiethnic survey of US adults after adjusting for main confounders.

\section{Methods}

2.1. Study Population. The Behavioral Risk Factor Surveillance System (BRFSS) is a federally funded, nationally representative survey of the civilian, noninstitutionalized, adult 
population aged 18 years or older. The survey is designed and conducted annually by the CDC in collaboration with the state health departments to monitor health-related behaviors and risk factors in the US population. The survey selects state-specific probability samples of households using a multistage cluster design to produce a nationally representative sample. The BRFSS uses random-digit dialing within blocks of telephone numbers to identify a probability sample of households with telephones in each state. In each household, one adult is randomly identified and interviewed. All 50 states, in addition to the District of Columbia, and the three US territories participated in the 2008 BRFSS. Detailed description of the BRFSS survey sample selection and study methodology are available online [12]. In 2008, the median cooperation rate was $75.0 \%$ and the median overall response rate was 53.3\% [12]. All participants provided informed consent and this study was approved by the West Virginia University Institutional Review Board.

To examine the association between insufficient rest/sleep and SRH, out of the 408,068 BRFSS participants who were aged $\geq 18$ years and not pregnant, we further excluded subjects with missing information on variables included in the multivariable model, including insufficient rest/sleep, SRH, body mass index, smoking, education, employment, physical activity, or physician-diagnosed cardiovascular disease. This resulted in 377,160 subjects with complete covariate data for the current analysis.

2.2. Main Outcome of Interest: SRH. In 2008, BRFSS included a new question on self-rated health, which asks, "Would you say that in general your health is excellent, very good, good, fair, poor, or do not know/not sure?" SRH is also termed "general health" because it refers to an individual's perception of his or her own health. The SRH continuum has been shown to be a reliable indicator of a person's objective or true health status [13]. A negative association between SRH and all-cause mortality has also been established [14].

2.3. Exposure Measurements. The 2008 BRFSS survey included the question, "During the past 30 days, for about how many days have you felt you did not get enough rest or sleep?" Data from all sites were aggregated, and the numbers of days of perceived insufficient rest or sleep were categorized into six groups as zero days, 1-6 days, 7-13 days, 14-20 days, 21-29 days, and 30 days. This question was previously tested and validated in the 2006 BRFSS survey in four states (Delaware, Hawaii, New York, and Rhode Island) [15]. Also, national estimates of insufficient rest or sleep according to these categories have been recently published by the CDC [16].

Age, gender, race/ethnicity, smoking status, alcohol intake, level of education, employment status, income, and physical activity were assessed using a standardized questionnaire. Individuals who had not smoked $\geq 100$ cigarettes in their lifetimes were classified as never smokers; those who had smoked $\geq 100$ cigarettes in their lifetimes were classified as former smokers or current smokers based on their response to the question on current smoking. Heavy alcohol drinking was defined as men who reported having more than 2 drinks per day, or more than 1 drink per day for women. Education was categorized into below high school, high school, or above high school education. Employment status was categorized as employed, unemployed, retired, unable to work, homemaker, or student. Body mass index (BMI) was categorized into $<25,25-29, \geq 30 \mathrm{~kg} / \mathrm{m}^{2}$. Subjects were classified as having no regular exercise if they reported not to be participating in any physical activities such as running, calisthenics, golf, gardening, or walking for exercise during the previous month. People married at the time of the survey were classified as married, while widows and divorcees were coded as unmarried. The annual household income variable was reported in quintiles as follows: less than $\$ 15,000, \$ 15,000-\$ 24,999, \$ 25,000-\$ 34,999, \$ 35,000$ $\$ 49,999, \$ 50,000$ or more. Depression (description used in BRFSS questionnaire: depression, major depression, dysthymia, or minor depression) and anxiety (description used in BRFSS questionnaire: acute stress disorder, anxiety, generalized anxiety disorder, obsessive-compulsive disorder, panic disorder, phobia, posttraumatic stress disorder, or social anxiety disorder) were categorized into dichotomous, yes/no variables.

2.4. Statistical Analysis. We examined the characteristics of the study sample by calculating variable frequencies. Perceived insufficient rest/sleep was categorized into six groups as zero days, 1-6 days, 7-13 days, 14-20 days, 21-29 days, and 30 days. Our single outcome of interest was SRH. We used logistic regression models to calculate odds ratio ((OR) $(95 \%$ confidence interval $(\mathrm{CI}))$ of the outcome of interest associated with increasing categories of insufficient rest/sleep, taking zero days of insufficient rest/sleep as the referent category. We used two logistic regression models: the unadjusted model and the multivariable model, adjusting for sex (men, women), age (years), race-ethnicity (non-Hispanic white, non-Hispanic black, Hispanic, other), education categories (<high school, high school, > high school), (employed, unemployed, retired, unable to work, homemaker, student), income category (quintiles), marital status (no, yes), smoker (never, former, current), heavy drinker (no, yes), no regular exercise (yes, no), BMI $\left(<25,25-29, \geq 30 \mathrm{~kg} / \mathrm{m}^{2}\right)$, CVD (no, yes), depression (no, yes), and anxiety (no, yes). Trends in the OR of the outcome across increasing insufficient rest/sleep category were determined by modeling these categories as an ordinal variable. Appropriate BRFSS survey weights that account for unequal probabilities of selection, oversampling, and nonresponse were applied for all analyses using SUDAAN (version 8.0; Research Triangle Institute, Research Triangle Park, NC) and SAS (version 9.2; SAS Institute, Cary, NC) software; SEs were estimated using the Taylor series linearization method.

\section{Results}

Table 1 presents characteristics of the study population. Nearly half of the study population were women, and about $17 \%$ were 65 years of age or older. Blacks comprised 9.7\% 
TABLE 1: Characteristics of the study sample $(n=377,160)$.

\begin{tabular}{|c|c|}
\hline Characteristic & Percentages \pm standard error $(\mathrm{SE})$ \\
\hline Women & $49.6 \pm .2$ \\
\hline 65 and over & $16.7 \pm .1$ \\
\hline \multicolumn{2}{|l|}{ Race/Ethnicity } \\
\hline Non-Hispanic white & $68.5 \pm .2$ \\
\hline Non-Hispanic black & $9.7 \pm .1$ \\
\hline Hispanic & $14.4 \pm .2$ \\
\hline Other & $6.8 \pm .1$ \\
\hline \multicolumn{2}{|l|}{ Education level } \\
\hline Below high school & $10.8 \pm .1$ \\
\hline High school & $28.6 \pm .2$ \\
\hline Above high school & $60.6 \pm .2$ \\
\hline \multicolumn{2}{|l|}{ Employment status } \\
\hline Employed & $60.8 \pm .2$ \\
\hline Unemployed & $6.0 \pm .1$ \\
\hline Retired & $15.8 \pm .1$ \\
\hline Unable to work & $5.0 \pm .1$ \\
\hline Homemaker & $7.6 \pm .1$ \\
\hline Student & $4.8 \pm .1$ \\
\hline \multicolumn{2}{|l|}{ Annual household income } \\
\hline$<\$ 15,000$ & $8.6 \pm .1$ \\
\hline$\$ 15,000-\$ 24,999$ & $13.7 \pm .1$ \\
\hline$\$ 25,000-\$ 34,999$ & $9.9 \pm .1$ \\
\hline$\$ 35,000-\$ 49,999$ & $13.0 \pm .1$ \\
\hline$\geq \$ 50,000$ & $44.3 \pm .2$ \\
\hline Married & $60.2 \pm .2$ \\
\hline \multicolumn{2}{|l|}{ Smoking } \\
\hline Never smoker & $18.7 \pm .1$ \\
\hline Former smoker & $24.6 \pm .1$ \\
\hline Current smoker & $56.8 \pm .2$ \\
\hline Heavy drinker & $5.3 \pm .1$ \\
\hline No regular exercise & $24.9 \pm .1$ \\
\hline \multicolumn{2}{|c|}{ Body mass index categories } \\
\hline Normal & $36.9 \pm .2$ \\
\hline Overweight or Obese & $63.1 \pm .2$ \\
\hline CVD & $8.2 \pm .1$ \\
\hline Depression & $1.4 \pm .03$ \\
\hline Anxiety & $1.1 \pm .03$ \\
\hline \multicolumn{2}{|l|}{ Insufficient rest/sleep } \\
\hline 0 days & $30.5 \pm .2$ \\
\hline $1-6$ days & $29.7 \pm .2$ \\
\hline $7-13$ days & $11.9 \pm .1$ \\
\hline 14-20 days & $13.4 \pm .1$ \\
\hline 21-29 days & $3.5 \pm .1$ \\
\hline 30 days & $11.0 \pm .1$ \\
\hline \multicolumn{2}{|l|}{ Self-rated health } \\
\hline Excellent & $20.8 \pm .1$ \\
\hline Very good & $33.1 \pm .2$ \\
\hline
\end{tabular}

of the study population, whereas Hispanics made up 14.4\%. Over $60 \%$ of study participants had above high school education and approximately the same percentage were employed.
TABLE 1: Continued.

\begin{tabular}{cc}
\hline Characteristic & Percentages \pm standard error $(\mathrm{SE})$ \\
\hline Good & $30.1 \pm .2$ \\
Fair & $11.7 \pm .1$ \\
Poor & $4.2 \pm .1$ \\
\hline
\end{tabular}

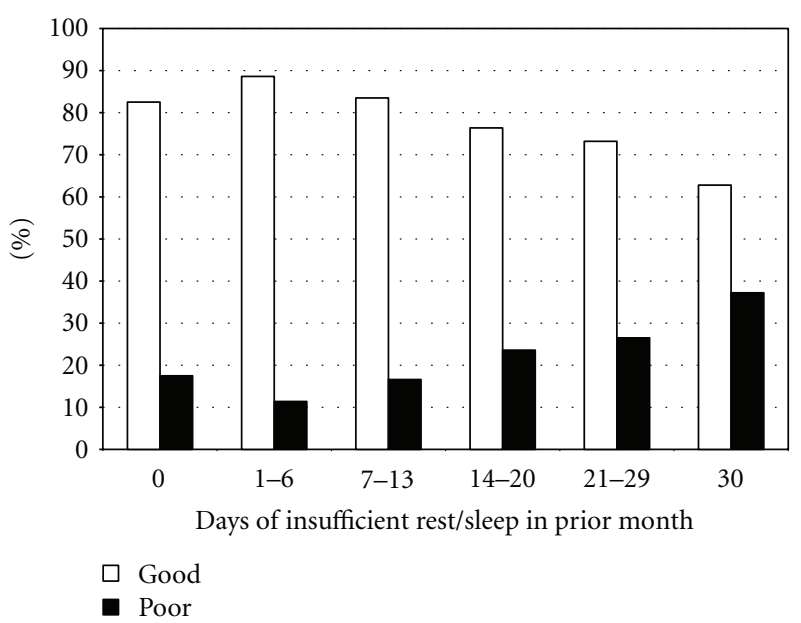

FIGURE 1: Insufficient rest/sleep by SRH. X-axis: Categories of insufficient rest or sleep in the past month. Y-axis: Weighted proportion (\%) of subjects within each insufficient rest/sleep category.

About 57\% were current smokers, 5.3\% were heavy drinkers, $63.1 \%$ were overweight or obese, and $25 \%$ reported no regular exercise. Finally, $8.6 \%$ of study subjects fell into the lowest income quintile and $8.2 \%$ reported having CVD.

Figure 1 presents the unadjusted distribution of SRH by categories of insufficient rest/sleep. Percentage of those with good SRH decreases across increasing categories of insufficient rest/sleep, whereas the opposite is true for individuals with poor SRH.

Table 2 presents the association between increasing categories of insufficient rest/sleep and poor SRH. We found that in the unadjusted model, a positive association between increasing categories of insufficient rest/sleep and poor SRH was evident only from $\geq 14$ days of insufficient rest/sleep. However, with multivariable adjustment, a linear dose-response association was evident $(P$-trend $<0.0001)$.

Tables 3, 4, 5, and 6 present the association between insufficient rest/sleep and poor SRH within stratified subgroups of gender, age, BMI, and race-ethnicity categories. We found that the positive association between insufficient rest/sleep and poor SRH persisted across all these stratified subgroups. Also, there was no statistically significant interaction between insufficient rest/sleep and SRH by age group, gender, or race/ethnciity (all $P$-interactions $>0.10$ ).

\section{Discussion}

In a multiethnic, representative sample of US adults, increasing categories of insufficient rest/sleep were shown to be positively associated with poor SRH overall and the association 
TABLE 2: Association between insufficient rest/sleep and poor SRH.

\begin{tabular}{|c|c|c|c|c|}
\hline $\begin{array}{l}\text { Categories of } \\
\text { insufficient rest/sleep }\end{array}$ & No. at risk & No. with poor SRH & $\begin{array}{c}\text { Unadjusted odds ratio } \\
\text { (95\% confidence intervals) }\end{array}$ & $\begin{array}{c}\text { Multivariable odds ratio } \\
(95 \% \text { confidence intervals) }\end{array}$ \\
\hline Whole cohort & 377160 & 70778 & & \\
\hline 0 days & 134596 & 23570 & 1 (referent) & 1 (referent) \\
\hline $1-6$ days & 106560 & 12190 & $0.64(0.61-.68)$ & $1.03(0.97-1.10)$ \\
\hline $7-13$ days & 39751 & 6580 & $0.89(0.83-.95)$ & $1.45(1.34-1.57)$ \\
\hline 14-20 days & 44781 & 10580 & $1.35(1.28-1.43)$ & $2.12(1.97-2.27)$ \\
\hline 21-29 days & 12152 & 3224 & $1.54(1.41-1.67)$ & $2.32(2.09-2.58)$ \\
\hline 30 days & 39320 & 14634 & $2.52(2.39-2.65)$ & $2.71(2.53-2.90)$ \\
\hline$P$-trend & & & $<0.0001$ & $<0.0001$ \\
\hline
\end{tabular}

* Adjusted for sex (men, women), age (years), race-ethnicity (non-Hispanic white, non-Hispanic black, Hispanic, other), education categories ( $<$ high school, high school, > high school), employment status (employed, unemployed, retired, unable to work, homemaker, student), income category (quintiles), marital status (no, yes), smoker (never, former, current), heavy drinker (no, yes), no regular exercise (yes, no), body mass index categories $\left(<25,25-29, \geq 30 \mathrm{~kg} / \mathrm{m}^{2}\right.$ ), CVD (no, yes), depression (no, yes), and anxiety (no, yes).

TABLE 3: Association between insufficient rest/sleep and poor SRH, by gender.

\begin{tabular}{|c|c|c|c|c|}
\hline $\begin{array}{l}\text { Categories of } \\
\text { insufficient rest/sleep }\end{array}$ & No. at risk & No. with poor SRH & $\begin{array}{l}\text { Unadjusted odds ratio } \\
\text { (95\% confidence intervals) }\end{array}$ & $\begin{array}{c}\text { Multivariable odds ratio } \\
\text { (95\% confidence intervals)* }\end{array}$ \\
\hline Men & 147300 & 26309 & & \\
\hline 0 days & 57456 & 9970 & 1 (referent) & 1 (referent) \\
\hline 1-6 days & 41088 & 4386 & $0.63(.58-.68)$ & $1.06(.97-1.17)$ \\
\hline 7-13 days & 14614 & 2175 & $0.82(.74-.91)$ & $1.41(1.24-1.61)$ \\
\hline 14-20 days & 15989 & 3557 & $1.23(1.13-1.35)$ & $2.02(1.81-2.25)$ \\
\hline 21-29 days & 4155 & 1011 & $1.50(1.30-1.73)$ & $2.42(2.03-2.90)$ \\
\hline 30 days & 13988 & 5210 & $2.53(2.33-2.75)$ & $2.70(2.42-3.00)$ \\
\hline$P$-trend & & & $<0.0001$ & $<0.0001$ \\
\hline Women & 229860 & 44469 & & \\
\hline 0 days & 77140 & 13600 & 1 (referent) & 1 (referent) \\
\hline $1-6$ days & 65472 & 7804 & $0.66(.62-.70)$ & $1.01(.94-1.09)$ \\
\hline 7-13 days & 25137 & 4405 & $0.94(.87-1.02)$ & $1.50(1.37-1.65)$ \\
\hline 14-20 days & 28.792 & 7023 & $1.45(1.35-1.55)$ & $2.21(2.03-2.42)$ \\
\hline 21-29 days & 7997 & 2213 & $1.55(1.40-1.71)$ & $2.26(2.01-2.54)$ \\
\hline 30 days & 25322 & 9424 & $2.48(2.33-2.65)$ & $2.72(2.50-2.96)$ \\
\hline$P$-trend & & & $<0.0001$ & $<0.0001$ \\
\hline
\end{tabular}

persisted when stratified by (1) gender, (2) age, (3) BMI, and (4) race-ethnicity. The positive association was present even when adjusting for confounding factors such as sex, age, race-ethnicity, education, employment status, income level, marital status, smoking, heavy drinking, exercise, BMI, depression, and anxiety. Our results indirectly suggest that poor SRH may be a mediator of the association between insufficient sleep and CVD and mortality [6, 7, 9, 14]. However, this hypothesis needs to be directly tested in a future study that simultaneously measures insufficient rest/sleep, $\mathrm{SRH}$, as well as CVD and mortality outcomes.

Internal validity for the detected association between perceived insufficient rest/sleep and poor SRH is high due to the magnitude of association, its independence from potential confounders such as sex, age, race-ethnicity, smoking, drinking, BMI, depression, and others. The presence of a positive dose-response trend and the persistence of the association in stratified analyses by subgroups of gender, age, BMI, and race-ethnicity suggest that our findings are not due to chance.

Only a few studies have examined the association between sleep and SRH and their results have been inconsistent. Segovia et al., in a study of 3,300 subjects from St. John's metropolitan area in Canada, found an association between sleep duration of less than 7 hours or greater than 8 hours and poor SRH [17]. However, the authors did not adjust for possible confounders such as ethnicity or depressive symptoms. Steptoe et al., in a study of more than 17,000 university 
TABLE 4: Association between insufficient rest/sleep and poor SRH, by age category.

\begin{tabular}{|c|c|c|c|c|}
\hline $\begin{array}{l}\text { Categories of } \\
\text { insufficient rest/sleep }\end{array}$ & No. at risk & No. with poor SRH & $\begin{array}{c}\text { Unadjusted odds ratio } \\
\text { (95\% confidence intervals) }\end{array}$ & $\begin{array}{c}\text { Multivariable odds ratio } \\
(95 \% \text { confidence intervals })^{*}\end{array}$ \\
\hline$<65$ years of age & 265278 & 40975 & & \\
\hline 0 days & 70920 & 8961 & 1 (referent) & 1 (referent) \\
\hline $1-6$ days & 81902 & 7186 & $0.74(.69-.79)$ & $1.03(.95-1.12)$ \\
\hline $7-13$ days & 32992 & 4416 & $1.04(.96-1.13)$ & $1.42(1.29-1.57)$ \\
\hline 14-20 days & 37806 & 7648 & $1.64(1.53-1.76)$ & $2.09(1.91-2.27)$ \\
\hline 21-29 days & 10431 & 2460 & $1.92(1.74-2.13)$ & $2.34(2.07-2.64)$ \\
\hline 30 days & 31227 & 10304 & $3.04(2.84-3.25)$ & $2.66(2.44-2.89)$ \\
\hline$P$-trend & & & $<0.0001$ & $<0.0001$ \\
\hline$\geq 65$ years of age & 111882 & 29803 & & \\
\hline 0 days & 63676 & 14609 & 1 (referent) & 1 (referent) \\
\hline 1-6 days & 24658 & 5004 & $0.90(.84-.96)$ & $1.06(.98-1.14)$ \\
\hline 7-13 days & 6759 & 2164 & $1.61(1.46-1.78)$ & $1.61(1.44-1.80)$ \\
\hline 14-20 days & 6975 & 2932 & $2.45(2.23-2.69)$ & $2.28(2.07-2.52)$ \\
\hline 21-29 days & 1721 & 764 & $2.48(2.07-2.98)$ & $2.19(1.79-2.69)$ \\
\hline 30 days & 8093 & 4330 & $3.85(3.54-4.19)$ & $2.96(2.68-3.26)$ \\
\hline$P$-trend & & & $<0.0001$ & $<0.0001$ \\
\hline
\end{tabular}

TABLE 5: Association between insufficient rest/sleep and poor SRH, by BMI.

\begin{tabular}{|c|c|c|c|c|}
\hline $\begin{array}{l}\text { Categories of } \\
\text { insufficient rest/sleep }\end{array}$ & No. at risk & No. with poor SRH & $\begin{array}{c}\text { Unadjusted odds ratio } \\
\text { (95\% confidence intervals) }\end{array}$ & $\begin{array}{c}\text { Multivariable odds ratio } \\
\text { (95\% confidence intervals)* }\end{array}$ \\
\hline $\begin{array}{l}\text { Normal weight } \\
\left(\mathrm{BMI}<25 \mathrm{~kg} / \mathrm{m}^{2}\right)\end{array}$ & 134529 & 19836 & & \\
\hline 0 days & 49373 & 7383 & 1 (referent) & 1 (referent) \\
\hline 1-6 days & 39532 & 3346 & $0.53(.48-.58)$ & $0.92(.82-1.02)$ \\
\hline $7-13$ days & 14100 & 1779 & $0.82(.73-.92)$ & $1.52(1.31-1.76)$ \\
\hline 14-20 days & 15100 & 2697 & $1.12(1.01-1.25)$ & $2.03(1.78-2.33)$ \\
\hline 21-29 days & 4033 & 822 & $1.32(1.13-1.55)$ & $2.27(1.88-2.73)$ \\
\hline 30 days & 12391 & 3809 & $2.40(2.18-2.66)$ & $2.85(2.50-3.24)$ \\
\hline$P$-trend & & & $<0.0001$ & $<0.0001$ \\
\hline $\begin{array}{l}\text { Overweight/Obese } \\
\left(\mathrm{BMI}>25 \mathrm{~kg} / \mathrm{m}^{2}\right)\end{array}$ & 242631 & 50942 & & \\
\hline 0 days & 85223 & 16187 & 1 (referent) & 1 (referent) \\
\hline 1-6 days & 67028 & 8844 & $0.70(.66-.75)$ & $1.09(1.01-1.17)$ \\
\hline $7-13$ days & 25651 & 4801 & $0.92(.85-.99)$ & $1.43(1.31-1.57)$ \\
\hline 14-20 days & 29681 & 7883 & $1.45(1.35-1.54)$ & $2.15(1.98-2.33)$ \\
\hline 21-29 days & 8119 & 2402 & $1.60(1.44-1.77)$ & $2.35(2.07-2.66)$ \\
\hline 30 days & 26929 & 10825 & $2.53(2.38-2.68)$ & $2.66(2.46-2.87)$ \\
\hline$P$-trend & & & $<0.0001$ & $<0.0001$ \\
\hline
\end{tabular}

students, found an association between only short sleep duration and poor SRH [18]. A study by Jean-Louis et al. found no association between sleep duration and poor SRH, but this study used a sample of only 273 California residents [19]. The current study found a significant association between insufficient sleep and poor SRH using a sample size of 377,160 , which is the largest study on this topic to date.
Also, due to our large sample size, we were able to conduct detailed subgroup analyses by gender, age, race-ethnicity, and $\mathrm{BMI}$ and confirm that the sleep-SRH association was present consistently within these subgroups.

Studies have reported that poor SRH is an independent predictor of mortality $[9,14]$. Studies have also shown that insufficient sleep predicts mortality [19]. Based on our 
TABLE 6: Association between insufficient rest/sleep and poor SRH, by race-ethnicity.

\begin{tabular}{|c|c|c|c|c|}
\hline $\begin{array}{l}\text { Categories of } \\
\text { insufficient rest/sleep }\end{array}$ & No. at risk & No. with poor SRH & $\begin{array}{l}\text { Unadjusted odds ratio } \\
\text { (95\% confidence intervals) }\end{array}$ & $\begin{array}{l}\text { Multivariable odds ratio } \\
\text { (95\% confidence intervals)* }\end{array}$ \\
\hline Non-Hispanic white & 299905 & 50401 & & \\
\hline 0 days & 106395 & 17161 & 1 (referent) & 1 (referent) \\
\hline $1-6$ days & 86387 & 8197 & $0.51(0.48-.53)$ & $0.89(0.84-.95)$ \\
\hline $7-13$ days & 32055 & 4565 & $0.76(0.71-.81)$ & $1.35(1.25-1.46)$ \\
\hline 14-20 days & 35899 & 7756 & $1.29(1.22-1.37)$ & $2.10(1.95-2.26)$ \\
\hline 21-29 days & 9884 & 2405 & $1.55(1.42-1.70)$ & $2.39(2.13-2.69)$ \\
\hline 30 days & 29285 & 10317 & $2.59(2.45-2.74)$ & $2.85(2.64-3.06)$ \\
\hline$P$-trend & & & $<0.0001$ & $<0.0001$ \\
\hline Non-Hispanic black & 28966 & 7727 & & \\
\hline 0 days & 9916 & 2312 & 1 (referent) & 1 (referent) \\
\hline $1-6$ days & 7822 & 1547 & $0.83(.72-.96)$ & $1.27(1.06-1.51)$ \\
\hline $7-13$ days & 3090 & 800 & $1.09(.90-1.32)$ & $1.56(1.25-1.95)$ \\
\hline 14-20 days & 3357 & 1123 & $1.67(1.40-2.00)$ & $2.53(2.04-3.14)$ \\
\hline 21-29 days & 889 & 324 & $1.75(1.29-2.39)$ & $2.88(2.08-3.99)$ \\
\hline 30 days & 3892 & 1621 & $2.39(2.05-2.79)$ & $2.75(2.28-3.32)$ \\
\hline$P$-trend & & & $<0.0001$ & $<0.0001$ \\
\hline Hispanic & 24810 & 7542 & & \\
\hline 0 days & 9707 & 2547 & 1 (referent) & 1 (referent) \\
\hline $1-6$ days & 6361 & 1560 & $1.10(.95-1.27)$ & $1.42(1.20-1.68)$ \\
\hline 7-13 days & 2305 & 739 & $1.38(1.12-1.69)$ & $1.82(1.45-2.28)$ \\
\hline 14-20 days & 2723 & 978 & $1.57(1.31-1.88)$ & $2.21(1.80-2.72)$ \\
\hline 21-29 days & 612 & 230 & $1.39(1.01-1.91)$ & $1.78(1.25-2.55)$ \\
\hline 30 days & 3102 & 1488 & $2.51(2.12-2.98)$ & $2.54(2.07-3.12)$ \\
\hline$P$-trend & & & $<0.0001$ & $<0.0001$ \\
\hline Other & 20556 & 4361 & & \\
\hline 0 days & 7280 & 1268 & 1 (referent) & 1 (referent) \\
\hline $1-6$ days & 5346 & 763 & $0.62(.49-.77)$ & $0.89(.69-1.13)$ \\
\hline $7-13$ days & 2051 & 408 & $1.17(.86-1.59)$ & $1.69(1.19-2.39)$ \\
\hline 14-20 days & 2515 & 631 & $1.53(1.21-1.93)$ & $1.85(1.39-2.47)$ \\
\hline 21-29 days & 664 & 236 & $2.19(1.56-3.06)$ & $2.55(1.72-3.77)$ \\
\hline 30 days & 2700 & 1055 & $2.66(2.13-3.33)$ & $2.42(1.84-3.19)$ \\
\hline$P$-trend & & & $<0.0001$ & $<0.0001$ \\
\hline
\end{tabular}

* Adjusted for sex (men, women), age (years), race-ethnicity (non-Hispanic white, non-Hispanic black, Hispanic, other), education categories ( $<$ high school, high school, >high school), (employed, unemployed, retired, unable to work, homemaker, student), income category (quintiles), marital status, smoker (never, former, current), heavy drinker (no, yes), no regular exercise (yes, no), body mass index categories $\left(<25,25-29, \geq 30 \mathrm{~kg} / \mathrm{m}^{2}\right)$, CVD (no, yes), depression (no, yes), and anxiety (no, yes).

findings, a corollary observation is that at least part of the association between poor SRH and mortality may be mediated by insufficient sleep. Also, insufficient rest/sleep may be related to sleep deficiency, which has been recently shown by Sorensen et al. to be related to multiple health outcomes as well as poor SRH [20]. The sleep deficiency/sleep adequacy constructs are similar to insufficient sleep but have historically been assessed using the Medical Outcomes Study sleep scale [21]. There is a need for future studies to examine the relation between similar measures of insufficient sleep and sleep adequacy and how they may jointly influence multiple health and well-being outcomes.

Strengths of the study include its large sample size, standardized data collection methods, and the availability of data on potentially confounding conditions such as BMI, depression, and anxiety. Also, the sample is nationally representative, with study samples from all 50 US states, the District of Columbia, and the three territories, men and women, and all race-ethnicities, which increases the study's generalizability. The first limitation of the study is its cross-sectional nature. Therefore, assertions about temporality or causation such as a sleep duration recommendations cannot be established here. Second, all BRFSS data are self-reported, which may lead to misclassification and therefore biased estimates. Third, the 2008 BRFSS did not include items on potential confounders such as shift work, sleep disorders, or relevant medication usage, which therefore were not controlled in this analysis. Fourth, the insufficient rest/sleep variable 
introduces a limitation due to subjectivity. Respondents may conceptualize the words "insufficient" and "rest" differently. Some respondents may have conceptualized true sleep when self-reporting, while others may have considered sleep plus rest, such as time spent watching television. The subjectivity makes comparison to studies of objective measures of insufficient sleep, including polysomnography, difficult. Fifth, the global SRH measure is also subjective and nonspecific, characteristics that may be viewed as limitations. However, the SRH measure's reliability and repeatability has been confirmed in multiple studies across several continents and various cultural settings $[13,22]$. Furthermore, SRH has the potential to capture dimensions of health that are not covered in traditional, objective clinical examinations, which are more likely to be focussed around a particular organ system (e.g., cardiovascular system or gastrointestinal system), and may also account for slowly progressing diseases that evade clinical diagnoses [23]. Sixth, the BRFSS survey did not collect information on habitual sleep duration. Therefore, unfortunately, we are not able to examine if the relationship between insufficient sleep and poor SRH is modified by habitual sleep duration. Finally, the insufficient rest/sleep item newly included in the 2008 BRFSS has not been widely used or tested and therefore its reliability and validity remains questionable. However, widely tested and validated sleep quality instruments such as the Pittsburgh Sleep Quality Index and the Epworth Sleepiness Scale do include similar items on perceived rest/sleep. Future studies examining the association between the insufficient rest/sleep BRFSS item and objectively measured sleep duration (e.g., actigraphy) are needed. Future research should also include longitudinal studies to establish causation potentially including sleep duration interventions [10].

In summary, in a multiethnic, nationally representative sample of US adults, increasing categories of perceived insufficient rest/sleep was found to be positively associated with poor SRH. This association persisted independent of sex, age, race-ethnicity, education, employment, income, marital status, smoking, heavy alcohol intake, lack of exercise, overweight/obese BMI, CVD, depression, and anxiety. Based on this study, self-reported insufficient rest/sleep appears to be a strong predictor of SRH.

\section{Conflict of Interests}

There are no conflicts of interest related to this paper.

\section{Acknowledgments}

This paper was made possible by Grant nos. $5 \mathrm{~T} 32$ HL090610-04 from the NIH/NHLBI and NIH/NIEHS Grants 1R03ES018888-01 and 5R03ES018888-02.

\section{References}

[1] O. M. Buxton and E. Marcelli, "Short and long sleep are positively associated with obesity, diabetes, hypertension, and cardiovascular disease among adults in the United States,"
Social Science and Medicine, vol. 71, no. 5, pp. 1027-1036, 2010.

[2] C. Sabanayagam and A. Shankar, "Sleep duration and cardiovascular disease: results from the National Health Interview Survey," Sleep, vol. 33, no. 8, pp. 1037-1042, 2010.

[3] A. Shankar, W. P. Koh, J. M. Yuan, H. P. Lee, and M. C. $\mathrm{Yu}$, "Sleep duration and coronary heart disease mortality among Chinese adults in Singapore: a population-based cohort study," American Journal of Epidemiology, vol. 168, no. 12, pp. 1367-1373, 2008.

[4] P. Heslop, G. D. Smith, C. Metcalfe, J. Macleod, and C. Hart, "Sleep duration and mortality: the effect of short or long sleep duration on cardiovascular and all-cause mortality in working men and women," Sleep Medicine, vol. 3, no. 4, pp. 305-314, 2002.

[5] A. Tamakoshi and Y. Ohno, "Self-reported sleep duration as a predictor of all-cause mortality: results from the JACC Study, Japan," Sleep, vol. 27, no. 1, pp. 51-54, 2004.

[6] B. Burström and P. Fredlund, "Self rated health: is it as good a predictor of subsequent mortality among adults in lower as well as in higher social classes?" Journal of Epidemiology and Community Health, vol. 55, no. 11, pp. 836-840, 2001.

[7] E. L. Idler and Y. Benyamini, "Self-rated health and mortality: a review of twenty-seven community studies," Journal of Health and Social Behavior, vol. 38, no. 1, pp. 21-37, 1997.

[8] L. Møller, T. S. Kristensen, and H. Hollnagel, "Self rated health as a predictor of coronary heart disease in Copenhagen, Denmark," Journal of Epidemiology and Community Health, vol. 50, no. 4, pp. 423-428, 1996.

[9] J. M. Mossey and E. Shapiro, "Self-rated health: a predictor of mortality among the elderly," American Journal of Public Health, vol. 72, no. 8, pp. 800-808, 1982.

[10] J. W. Newbury, J. E. Marley, and J. J. Beilby, "A randomised controlled trial of the outcome of health assessment of people aged 75 years and over," Medical Journal of Australia, vol. 175, no. 2, pp. 104-107, 2001.

[11] Committee on Quality of Health Care in America, Institute of Medicine, Crossing the Quality Chasm: A New Health System for the 21st Century, National Academy Press, Washington, DC, USA, 2001.

[12] Centers for Disease Control and Prevention (CDC), "BRFSS overview," 2008, http://www.cdc.gov/brfss/technical_infodata/ surveydata/2008/overview_08.rtf.

[13] K. Manderbacka, E. Lahelma, and P. Martikainen, "Examining the continuity of self-rated health," International Journal of Epidemiology, vol. 27, no. 2, pp. 208-213, 1998.

[14] S. Miilunpalo, I. Vuori, P. Oja, M. Pasanen, and H. Urponen, "Self-rated health status as a health measure: the predictive value of self-reported health status on the use of physician services and on mortality in the working-age population," Journal of Clinical Epidemiology, vol. 50, no. 5, pp. 517-528, 1997.

[15] Centers for Disease Control and Prevention (CDC), "Perceived insufficient rest or sleep-four states, 2006," Morbidity and Mortality Weekly Report, vol. 57, pp. 200-203, 2008.

[16] Centers for Disease Control and Prevention (CDC), "Perceived insufficient rest or sleep among adults-United States, 2008," Journal of the American Medical Association, vol. 302, pp. 2532-2539, 2009.

[17] J. Segovia, R. F. Bartlett, and A. C. Edwards, "The association between self-assessed health status and individual health practices," Canadian Journal of Public Health, vol. 80, no. 1, pp. 32-37, 1989. 
[18] A. Steptoe, V. Peacey, and J. Wardle, "Sleep duration and health in young adults," Archives of Internal Medicine, vol. 166, no. 16, pp. 1689-1692, 2006.

[19] D. F. Kripke, L. Garfinkel, D. L. Wingard, M. R. Klauber, and M. R. Marler, "Mortality associated with sleep duration and insomnia," Archives of General Psychiatry, vol. 59, no. 2, pp. 131-136, 2002.

[20] G. Sorensen, A. M. Stoddard, S. Stoffel et al., "The role of the work context in multiple wellness outcomes for hospital patient care workers," Journal of Occupational and Environmental Medicine, vol. 53, no. 8, pp. 899-910, 2011.

[21] R. D. Hays, S. A. Martin, A. M. Sesti, and K. L. Spritzer, "Psychometric properties of the Medical Outcomes Study Sleep measure," Sleep Medicine, vol. 6, no. 1, pp. 41-44, 2005.

[22] T. F. Crossley and S. Kennedy, "The reliability of self-assessed health status," Journal of Health Economics, vol. 21, no. 4, pp. 643-658, 2002.

[23] M. Jylhä, "What is self-rated health and why does it predict mortality? Towards a unified conceptual model," Social Science and Medicine, vol. 69, no. 3, pp. 307-316, 2009. 


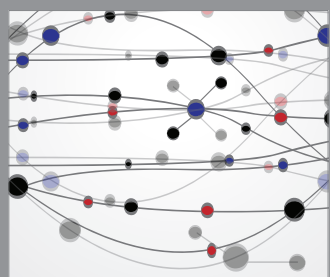

The Scientific World Journal
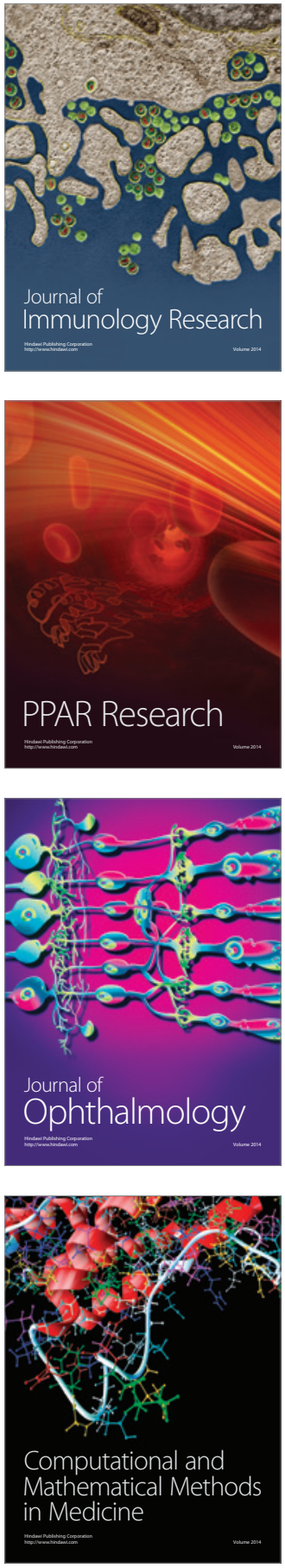

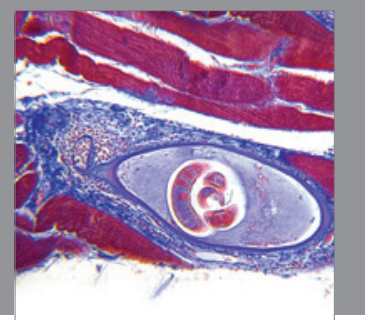

Gastroenterology

Research and Practice
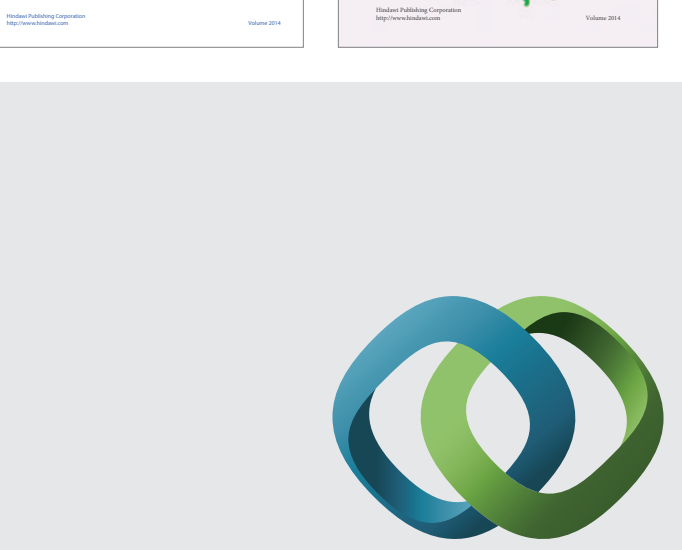

\section{Hindawi}

Submit your manuscripts at

http://www.hindawi.com
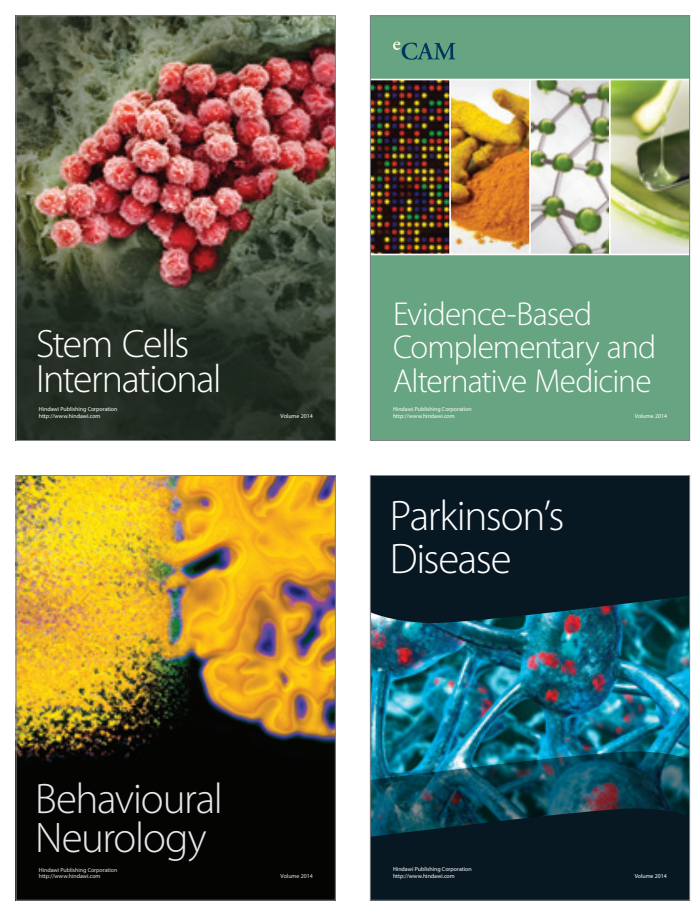

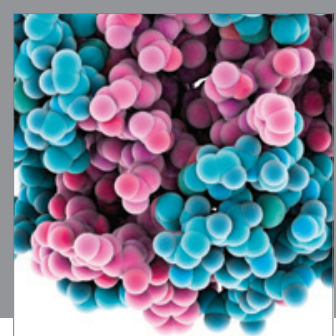

Journal of
Diabetes Research

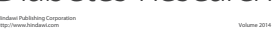

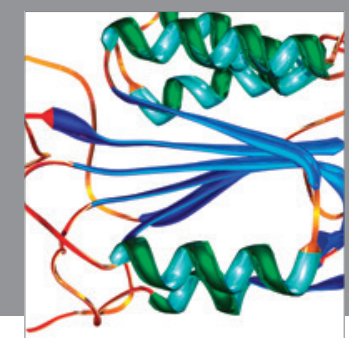

Disease Markers
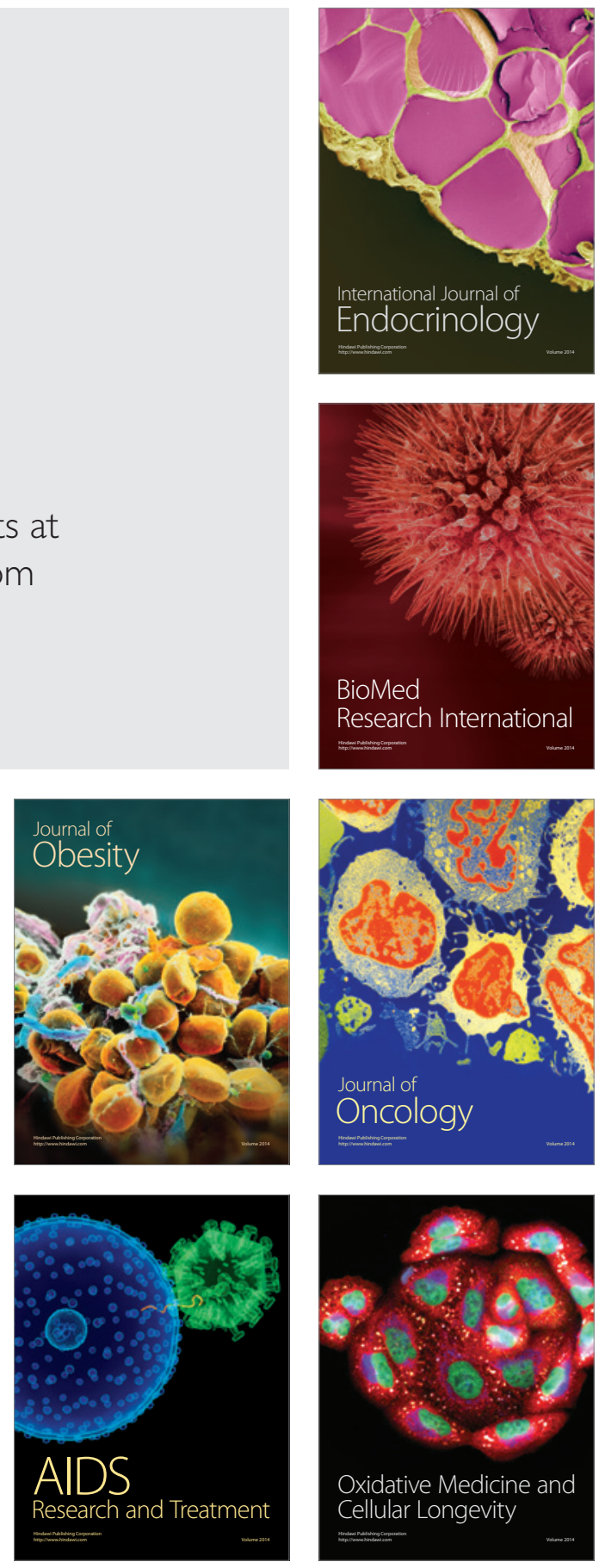